\title{
Stress Response in Swedish Ambulance Personnel Evaluated by Trier Social Stress Test
}

\author{
Kåre Karlsson $^{1,2,3}$, Patrik Persson Niemelä ${ }^{1,2}$, Anders Jonsson ${ }^{4,5}$, Carl-Johan Törnhage ${ }^{6,7, *}$ \\ ${ }^{1}$ Ambulance Services, Skaraborg Hospital, Skovde, Sweden \\ ${ }^{2}$ The Research Fund at Skaraborg Hospital, Skovde, Sweden \\ ${ }^{3}$ School of Health and Welfare, University of Jonkoping, Jonkoping, Sweden \\ ${ }^{4}$ School of Health Sciences, University of Borås, Borås, Sweden \\ ${ }^{5}$ Centre for Defence Medicine, The Swedish Armed Forces, West Frolunda, Sweden \\ ${ }^{6}$ Department of Paediatrics, Skaraborg Hospital, Skovde, Sweden \\ ${ }^{7}$ Sahlgrenska Academy, University of Gothenburg, Gothenburg, Sweden \\ Email address: \\ carl-johan_tornhage@hotmail.com (Carl-Johan T.) \\ ${ }^{*}$ Corresponding author
}

\section{To cite this article:}

Kåre Karlsson, Patrik Persson Niemelä, Anders Jonsson, Carl-Johan Törnhage. Stress Response in Swedish Ambulance Personnel Evaluated by Trier Social Stress Test. Journal of Health and Environmental Research. Vol. 5, No. 1, 2019, pp. 14-23. doi: 10.11648/j.jher.20190501.13

Received: January 7, 2019; Accepted: March 2, 2019; Published: March 19, 2019

\begin{abstract}
According to previous research, ambulance personnel often consider themselves as healthy, but at the same time several studies show that they suffer from several stress-related illnesses, take early retirement and even suffer early death. The aim of this study was to explore mental stress during the Trier Social Stress Test. Questions were whether heart rate measurement could replace cortisol concentration in saliva as an indicator of stress and if there were differences between genders. During 20 Trier Social Stress Tests heart rate and salivary cortisol concentrations were measured. Heart rate was measured every 15 seconds and salivary cortisol was collected at seven occasions. Fourteen men and six women (sixteen ambulance nurses and four paramedics) participated. A questionnaire with background data was collected. Statistical analysis used was non-parametric tests to adjust for misalignment. During the Trier Social Stress Test women had their highest salivary cortisol concentration before start of test while the maximum values for men were 10 to 20 minutes after start. In contrast, there was no difference in heart rhythm before, during and after test between genders. No correlation between heart rate and salivary cortisol was found. There was no significant difference in stress response according to personnel's age or level of education. Women and men exhibit different hormonal stress responses when it comes to performing unfamiliar actions, something that has not been seen before. Since no correlation could be seen between heart rate and salivary cortisol concentration they cannot replace each other as indicators of stress.
\end{abstract}

Keywords: Ambulance Personnel, Heart Rate, Salivary Cortisol, Stress, Trier Social Stress Test

\section{Introduction}

The ambulance service aims to take care of sick or injured people indoors and outdoors, during summer and winter, 24 hours a day, and sometimes under additional pressure. The teams' compositions vary in terms of physical abilities, age, height, gender and education. The Swedish ambulance service has to follow the regulations of the National Board of Health [1] stating that each team must consist of at least one registered nurse who is medically responsible for the patient and for the administration of drugs. The other team member can be either a nurse or a nursing assistant, called paramedic.

\subsection{Ambulance Personnel}

Ambulance personnel consider themselves healthy [2], but in spite of this they state various medical symptoms. A study found that $98 \%$ felt that they had good health, while at the same time $69 \%$ stated that they had at least one medical 
problem, and one third reported emotional problems that sometimes influenced their work [3]. Previous studies show that nearly one third of the paramedics suffer symptoms of stress, burnout, post-traumatic stress disorder (PTSD) or other psychosomatic illnesses, which is a higher proportion than for many other professions [4, 5]. Sterud et.al. [6] showed that paramedics are at increased risk of injury at work and also risk increased rates of mental health problems. They could also see an increased incidence of early retirement and a risk of early death often linked to health problems. Another study showed that paramedics run more than twice as high a risk of work-related death compared to other workers [7]

It has been established that the nursing profession in general generates an increased risk of stress [8], but the underlying causes are more uncertain. That emergency nurses experience higher stress than general ward nurses was seen in a study [9]. In spite of this, many ambulance personnel look forward to the really serious alarms [10].

Earlier research has stated that an increased risk of higher morbidity such as cardiovascular disease and stroke might be linked to occupational stress and job strain $[11,12]$ The link between cancer and job strain is weaker [13]. Previous research has demonstrated that ambulance personnel accumulate a number of risk factors including both acute and chronic stress [14] but there is sparse research about the actual causes.

\subsection{Stress Consequences}

Perceived job stress can be related to patient care in the ambulance, and this stress is also noticeable the following day at home. Perceived stress on a work- free day also influences the perceived stress associated with patient care the next day [15]. This stress is also demonstrated physiologically in higher cortisol concentration in personnel the morning prior to a shift in an emergency ambulance compared to a transport ambulance. This was interpreted as an expectation of higher demands during work in the emergency ambulance [16].

Work-related stress in emergency care may lead to poorer patient care and medical mistakes [17]. Little opportunity to influence a demanding job situation may lead to increased stress and consequently worse patient safety [18]. This was confirmed in simulated high-stress patient cases where it was found that the risks of mistakes were greater, taking the form of pharmaceutically miscalculated doses [19], worse clinical performance and inadequate documentation [20].

\subsection{Theoretical Background}

Health and disease is complex and multifaceted and, in order to get an overall grasp on health-disease-care, a multidisciplinary approach is required [21]. In our specific field, it is primarily medicine and applied care that is the theoretical basis. Medical biological science and theory are traditionally based on health being the absence of disease [22]. Another way of thinking is to try to identify biological and psychological conditions and to find out if these are desirable or not for the individual. The question then is whether we describe a state that the individual is in or one that we want the person to be in [23]? Caring science can be regarded as a supplement and extension to the bio-medical paradigm. A central core is that health is not the absence of disease without the patient's experience of illness, that is, you can have a disease without experiencing you sick [24]. The perception of health is not only sovereignty from illness but also something that involves the whole being and is formed by her life experience. Health is individual and important to understand as a vital force that is important to promote [25, 26]. The experience of health is therefore unique and must be seen as multidimensional and comparative [27]. Stress and stress reactions are a normal reaction meant to protect the individual from dangers. A repetitive work-related stress, on the other hand, can be a contributing factor to diseases. This study rests primarily on the biomedical foundation since there are biological markers that form the basis of the study.

\subsection{Methods to Measure Stress}

Salivary cortisol is a simple and well-established method to measure stress and the hypothalamic-hypophyse-adrenal axis, and it correlates well with the serum concentration of cortisol [28, 29]. Heart rate can serve as a clinical indicator of stress and is easily measured with a heart rate monitor [30]. The Trier Social Stress Test (TSST) is a proven standardized psychological stress test and well-developed method to induce physiological responses in laboratory settings [31]. The method has proven to be valid in different contexts [3234]. In this study the participants had to prepare for an unknown task, in this case an employment interview for 10 minutes. This was followed by an unprepared calculation exercise for 5 minutes. This was to resemble an ambulance mission where the staff in connection to the assignment usually gets limited information to prepare from. Like TSST, situations and information often change quickly in the short time staff has to take care of the patient, which often leads to rapid changes in decision making in a sometimes pressured situation.

\subsection{Purpose}

The primary purpose was to explore the mental stress of ambulance personnel during the TSST and to explore whether gender, experience or education affected the results. The secondary purpose was to evaluate whether the measurement of heart rate was consistent and comparable with cortisol as an indicator of stress and thus whether heart rate measurement could replace cortisol as a more reliable easy way of registration during work.

\subsection{Hypotheses}

1. The TSST causes mental stress among ambulance personnel

2. Gender, professional experience, education and age affect the stress response 
3. Heart rate can replace salivary cortisol as a measurement of stress

\section{Methods}

\subsection{Study Design and Data Source}

The study was conducted in an ambulance organization in southern Sweden. This organization consists of twelve ambulances on duty for 24-hours shifts and eight ambulances on duty only during daytime shifts, $7.45 \mathrm{am}-5 \mathrm{pm}$ or 7.45 am-11 pm. The total number of employees at the start of the investigation was 164 of whom 129 were nurses and 35 were paramedics.

A request for participation was sent to all staff members by mail and returned by those who agreed to participate. The only exclusion criterion was if they were on medication that affected their heart rate and / or cortisol concentration.

Participants wore heart rate watches of the brand Polar RS $400^{\mathrm{TM}}$ (Polar Electro, Bromma, Sweden) that logged the heart rate every 15 seconds during the time span when salivary cortisol was collected. All participants were given instructions on how to handle the equipment.

\subsection{TSST}

Salivary cortisol was collected using neutral cotton-based Salivette $^{\mathrm{TM}}$ (Sarstedt, Numrecht, Germany) tubes. During the test, saliva samples were taken ten minutes before the start of the test and at 1, 10, 20, 30, 45 and 60 minutes after the end of the test. To minimize impact on the test result participants were told to avoid smoking, using snuff, brushing teeth, and drinking coffee before data collection.

\subsection{Perceptions of Stress, Anxiety and Emotions During and After Test}

These factors were also measured from start to 60 minutes after the end of the test. Perceived Stress and anxiety levels were given on a continuous scale, 0 to 100 . "0" was defined as no stress or anxiety and " 100 " as extreme perceived stress and anxiety. In opposite, emotionally feelings were defined as " 100 " feel very well to supreme and " 0 " feel awful, worst emotions.

\subsection{Participants}

The total ambulance group consisted of 164 people of whom 35 were paramedics and 129 nurses. The 99 men were from 31 to 62 years old (median $=42$ years) and the 65 women were from 27 to 56 years old (median $=41$ years). All employees were informed about the study at workplace meetings and given the opportunity to participate. 38 agreed to participate, among three were excluded because of medication. 20 were randomly selected from this group by being drawn by a nonparticipating individual. One participant had to drop out immediately due to an injury and was randomly replaced. Of the participants, 14 were men and 6 women. The median age was 43 years (32-53) for men and
41 years (31-44) for women. Ten men and 6 women were nurses. All participants had a permanent employment and had undergone tests and interviews before employment to check the suitability for the profession. All employees perform regular simulation tests to adapt to specific situations to be rationale in their work.

\subsection{Procedure-Randomization}

All participants were given both oral and written information. Information was also given that no participant would have access to their personal results. After their written consent to participate in the study, the participants were coded randomly from a numbered code list by a nonparticipating individual.

\subsection{Study Period and Sequencing the TSST}

TSST was carried out on 20 different days. Time for each participant was randomized according to 20 days when the researcher (C-JT) and the different participants could meet each other. Only one test per participant was carried out since the element of surprise in the test could not be repeated. All the TSSTs were performed in the same room on a work freeday. The participant's used their own cars for transportation to the test site, the journeys being between 15-80 minutes. All tests began between 8.03 to 8.50 am and the last took place $10.24 \mathrm{am}$. The participants had woken between 04.00 $06.55 \mathrm{am}$, mostly between $06.00-06.50 \mathrm{am}$. The TSSTs were supervised by at least one of the researchers.

\subsection{Key Independent and Outcome Variables}

Outcome variables were salivary cortisol concentration, heart rate and stress perception, anxiety and emotional insecurity. The independent variables were gender, experience, education and age.

\subsection{Collection and Analysis of Salivary Cortisol Samples}

All participants followed a well described technique for the collection of salivary cortisol samples [28]. Briefly, a neutral cotton-based swab, Salivette ${ }^{\mathrm{TM}}$ (Sarstedt, Numrecht, Germany), was chewed for about 2 minutes and then placed in its double lumen tube. The tube was submitted to the laboratory and refrigerated until it was centrifuged at 3000 rpm for 10 minutes at room temperature. After this the saliva was frozen to between -20 and $-80^{\circ} \mathrm{C}$ prior to analysis. All the samples were analyzed on the same occasion as double tests. A commercial RIA-based method for salivary cortisol was used, Spectria ${ }^{\mathrm{TM}}$ Cortisol $\mathrm{I}^{125}$ (Orion Diagnostica, Landskrona, Sweden). The lower limit for detection is 0.5 $\mathrm{nmol} / \mathrm{l}$ and upper limit $100 \mathrm{nmol} / \mathrm{L}$. The intra-assay precision is about $5 \%$ and inter-assay precision is about $6-7 \%(\mathrm{CV})$. Samples were analysed in a MultiGamma 1261 (Wallac).

\subsection{Statistical Analysis}

The data were entered into a Microsoft Excel spreadsheet Version 2013 (Microsoft Corporation, Redmond, 
Washington, USA). STATISTICA version 10.0 (Stat Soft, Uppsala, Sweden) and SPSS version 18.0 (SPSS Inc. Chicago, IL, USA) were used for statistical calculations. Non-parametric tests were used to adjust for misalignment. The Mann-Whitney U test and the Kruskal-Wallis one-way analysis of variance were used to compare the groups. Statistical significance was considered to be present if a 2sided exact $\mathrm{p}$-value was $<0.05$.

\subsection{Presentation}

The total secretion of cortisol during TSST and recovery time was calculated as the area under the curve, AUC from zero level $\left(\mathrm{AUC}_{0}=\mathrm{AUC}_{\mathrm{G}}\right)$ according to previous research $[36,37]$.

\section{Results}

\subsection{Characteristics of Study Subjects}

Comparisons of the characteristics of the participants' versus non-participants' gender, age or education showed no significant differences, Table 1.

Table 1. Characteristics of the study population.

\begin{tabular}{|c|c|c|c|c|c|}
\hline \multirow{2}{*}{ Population } & \multicolumn{2}{|c|}{ Original } & \multicolumn{2}{|l|}{ Study } & \multirow{2}{*}{ p-value ${ }^{1}$} \\
\hline & $\mathbf{n}$ & $\%$ & $\mathbf{n}$ & $\%$ & \\
\hline Number & 164 & 100 & 20 & 12 & - \\
\hline Median age (years) [Male/Female] & $42 / 43$ & - & $41 / 41$ & - & 0.93 \\
\hline Education [Nurse/Paramedics] & $129 / 35$ & $78 / 22$ & $16 / 4$ & $80 / 20$ & 0.95 \\
\hline
\end{tabular}

Comparisons between participants and all invited personnel. Number and percentage of participants are given in relation to gender, age and education. ${ }^{1}$ Statistical analysis; Mann-Whitney U test.

\subsection{TSST}

The exact results for heart rate and salivary cortisol levels before, during and one hour after the end of the TSST are shown in Table 2.

Table 2. Salivary cortisol concentration and heart rate at Triers' Social Stress Test according to genders.

\begin{tabular}{|c|c|c|c|c|c|c|c|}
\hline \multirow{2}{*}{ Time $^{1}$} & & \multicolumn{3}{|c|}{ Salivary cortisol $(\mathrm{nmoL} / \mathrm{L})$} & \multicolumn{3}{|c|}{ Heart rate (bpm) } \\
\hline & & Women $n=6$ & Men $n=14$ & p-value & Women $n=6$ & Men $n=14$ & p-value \\
\hline \multirow{2}{*}{-10} & Median & 30.7 & 20.6 & \multirow{2}{*}{$0.03 *$} & 73.5 & 73.0 & \multirow{2}{*}{ n.s } \\
\hline & 10 - 90 Perc. & 20.8 to 43.8 & 15.3 to 32.6 & & 61.6 to 88.2 & 60.7 to 93.1 & \\
\hline \multicolumn{8}{|c|}{ Triers Social Stress Test } \\
\hline \multirow{2}{*}{+1} & Median & 27.7 & 25.1 & \multirow{2}{*}{ n.s } & 73.0 & 67.0 & \multirow{2}{*}{ n.s } \\
\hline & 10 - 90 Perc. & 21.7 to 33.2 & 14.2 to 40.8 & & 52.5 to 82.8 & 56.4 to 84.3 & \\
\hline \multirow{2}{*}{+10} & Median & 26.9 & 28.3 & \multirow{2}{*}{ n.s } & 64.0 & 64.0 & \multirow{2}{*}{ n.s } \\
\hline & 10 - 90 Perc. & 21.3 to 32.7 & 14.1 to 45.5 & & 48.1 to 84.0 & 53.2 to 78.7 & \\
\hline \multirow{2}{*}{+20} & Median & 25.8 & 26.0 & \multirow{2}{*}{ n.s } & 64.0 & 60.5 & \multirow{2}{*}{ n.s } \\
\hline & 10 - 90 Perc. & 19.3 to 35.5 & 12.9 to 44.1 & & 49.0 to 81.2 & 50.4 to 76.1 & \\
\hline \multirow{2}{*}{+30} & Median & 24.0 & 24.8 & \multirow{2}{*}{ n.s } & 65.0 & 62.5 & \multirow{2}{*}{ n.s } \\
\hline & 10 - 90 Perc. & 17.3 to 30.7 & 12.3 to 35.7 & & 54.7 to 77.0 & 49.9 to 75.3 & \\
\hline \multirow{2}{*}{+45} & Median & 20.1 & 19.9 & \multirow{2}{*}{ n.s } & 56.5 & 61.0 & \multirow{2}{*}{ n.s } \\
\hline & 10 - 90 Perc. & 14.5 to 27.3 & 13.0 to 26.3 & & 46.0 to 77.5 & 51.8 to 74.5 & \\
\hline \multirow{2}{*}{+60} & Median & 18.1 & 17.0 & \multirow{2}{*}{ n.s } & 61.0 & 62.0 & \multirow{2}{*}{ n.s } \\
\hline & 10 - 90 Perc. & 13.7 to 24.6 & 12.7 to 24.5 & & 49.5 to 81.3 & 51.6 to 74.7 & \\
\hline
\end{tabular}

${ }^{1}$ Minutes before $(-)$ and after $(+)$ performed TSST. Perc. $=$ Percentile, Bpm=beat per minute. Statistical analysis; MWU test. n.s=non-significant. * significant difference.

\subsection{Heart Rate Variability (HRV)}

HRV before and after the test are shown in Table 2 and Figure 1. Men and women showed the same pattern with a higher heart rate during expectation than in the period immediately after the test and during recovery. Comparison of heart rate during the TSST, revealed no significant differences between genders. 

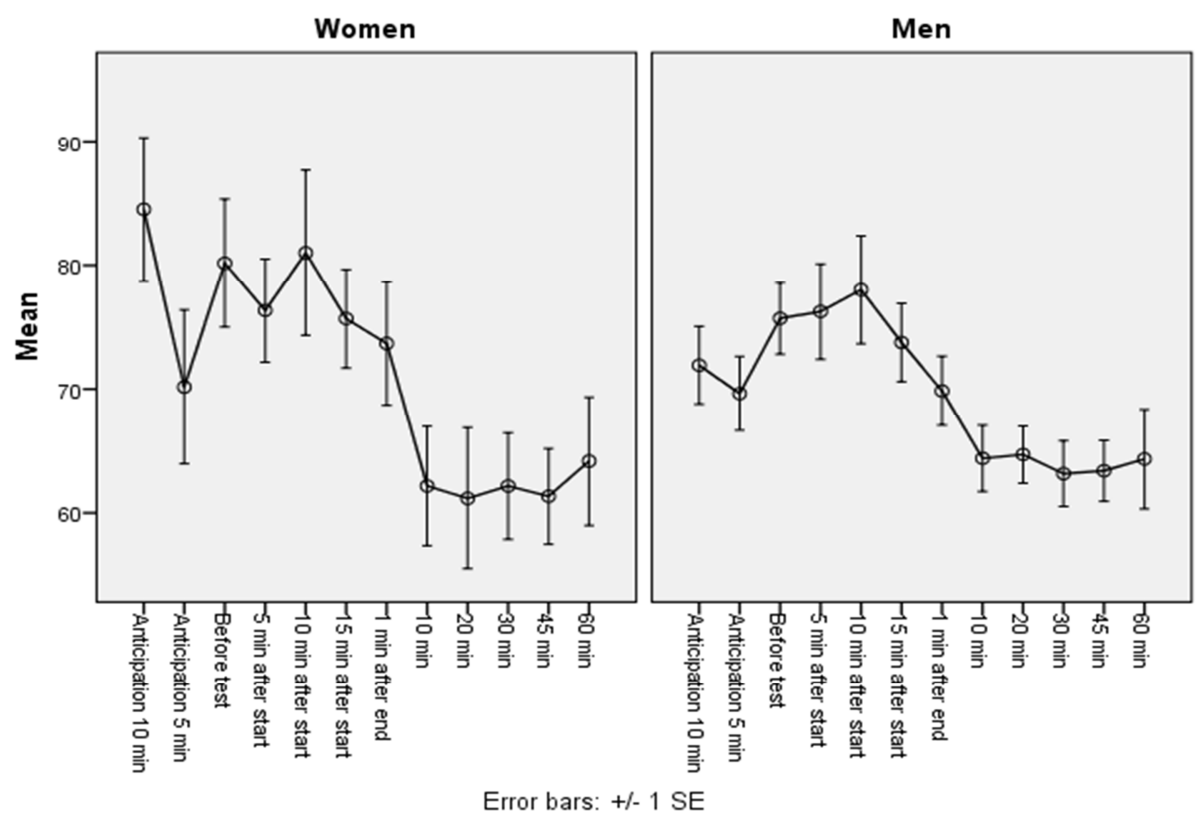

Figure 1. Heart rate (bpm) before, during and after Triers Social Stress Test (TSST) in 6 female and 14 male ambulance personnel. Values are given as mean $+/-1$ standard error mean (SE). There were no gender differences.

\subsection{Saliva Cortisol Concentration}

There were different patterns between women and men regarding salivary cortisol concentration. In five out of six women the highest concentration was during expectation

Women $n=6$

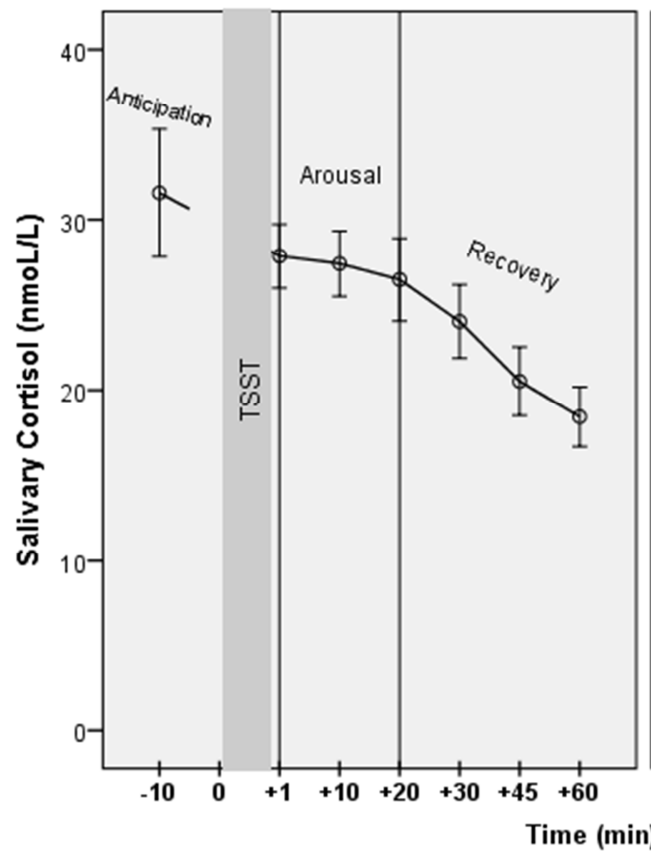

before the start of the test. In contrast, men's' top was at 10 to 20 minutes after completing the TSST, Figure 2. The difference in pattern between genders was significant, $\mathrm{p}=$ 0.03 , Table 2 .

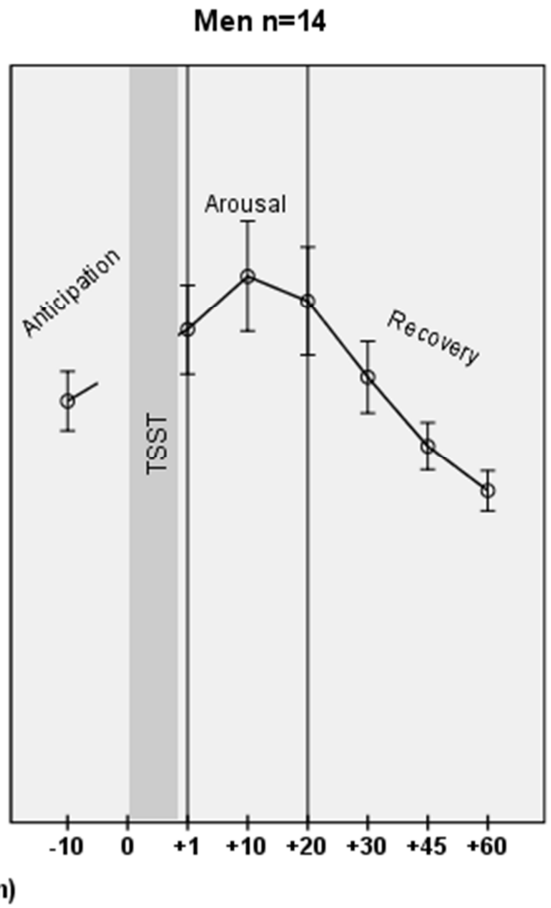

Figure 2. Salivary cortisol concentrations during anticipation, arousal and recovery of Triers Social Stress Test (TSST). Comparison of female and male ambulance personnel. Values are given as mean $+/-1$ standard error of mean. There was a significant difference between gender during the anticipation, $p=0.03$.

The absolute AUC calculated from ground ( $\mathrm{AUC}_{0}$ ) and relative changes AUC increase $\left(\mathrm{AUC}_{\mathrm{I}}\right)$ in salivary cortisol and heart rate are shown in Table 3. There was a significant decrease in salivary cortisol secretion in women in contrast to a minor increase in men. During the first phase - the anticipation - the difference between genders was significant, $\mathrm{p}=0.01$. The relative changed secretion of cortisol during the TSST including all three phases, $\mathrm{AUC}_{\mathrm{I}}$ tot, was different between gender, $\mathrm{p}=0.04$. 
Table 3. Total salivary cortisol secretion and heart rate*time product (Area under curve $=A U C$ ) during Trier's Social Stress Test.

\begin{tabular}{|c|c|c|c|}
\hline AUC Salivary cortisol Mean (SD) & Women $n=6$ & Men n=14 & p-value \\
\hline $\mathrm{AUC}_{0}$ part 1 & $327(74)$ & $271(85)$ & 0.22 \\
\hline $\mathrm{AUC}_{0}$ part 2 & $518(82)$ & $546(217)$ & 0.87 \\
\hline $\mathrm{AUC}_{0}$ part 3 & $878(200)$ & $872(259)$ & 1.00 \\
\hline $\mathrm{AUC}_{0}$ tot & $1723(299)$ & $1689(548)$ & 0.74 \\
\hline $\mathrm{AUC}_{\mathrm{I}}$ part 1 & $-20(31)$ & $23(32)$ & $0.01 *$ \\
\hline $\mathrm{AUC}_{\mathrm{I}}$ part 2 & $-11(74)$ & $38(54)$ & 0.06 \\
\hline $\mathrm{AUC}_{\mathrm{I}}$ part 3 & $-180(56)$ & $-263(251)$ & 0.74 \\
\hline $\mathrm{AUC}_{\mathrm{I}}$ tot & $-486(648)$ & $112(290)$ & $0.04^{*}$ \\
\hline \multicolumn{4}{|l|}{ AUC Heart rate Mean (SD) } \\
\hline $\mathrm{AUC}_{0}$ part 1 & 799 (119) & $784(116)$ & 0.87 \\
\hline $\mathrm{AUC}_{0}$ part 2 & $1248(223)$ & $1226(184)$ & 0.68 \\
\hline $\mathrm{AUC}_{0}$ part 3 & $2507(393)$ & $2491(374)$ & 0.68 \\
\hline $\mathrm{AUC}_{0}$ tot & $4555(720)$ & $4501(658)$ & 0.48 \\
\hline $\mathrm{AUC}_{\mathrm{I}}$ part 1 & $-16(28)$ & $-28(26)$ & 0.38 \\
\hline $\mathrm{AUC}_{\mathrm{I}}$ part 2 & $-104(110)$ & $-80(59)$ & 0.65 \\
\hline $\mathrm{AUC}_{\mathrm{I}}$ part 3 & $-40(93)$ & $-6(100)$ & 0.56 \\
\hline $\mathrm{AUC}_{\mathrm{I}}$ tot & $-637(270)$ & $-669(278)$ & 0.74 \\
\hline
\end{tabular}

Part $1=$ Anticipation (between $-10 \mathrm{~min}$ and $+1 \mathrm{~min}$ )

Part $2=$ Arousal (between +1 and +20 )

Part $3=$ Recovery (between +20 and +60 )

$\mathrm{AUC}_{0}=\mathrm{AUC}$ calculated from ground i.e. 0

$\mathrm{AUC}_{\mathrm{I}}=\mathrm{AUC}$ increase (calculated from first observation).

Values are given as mean and standard deviation (SD). Statistical analysis, MWU test, ${ }^{*} \mathrm{p}<0.05$.

\subsection{Perceptions of Stress, Anxiety and Emotions Before, During and After TSST}

The perceived stress, anxiety and emotional feelings were normal before start and changed fast after the beginning of the test and were recovered soon after end of test. This pattern of response correlates well with the heart rate response. For details see figure 3.

Stress, anxiety and emotions before, during and after TSST.

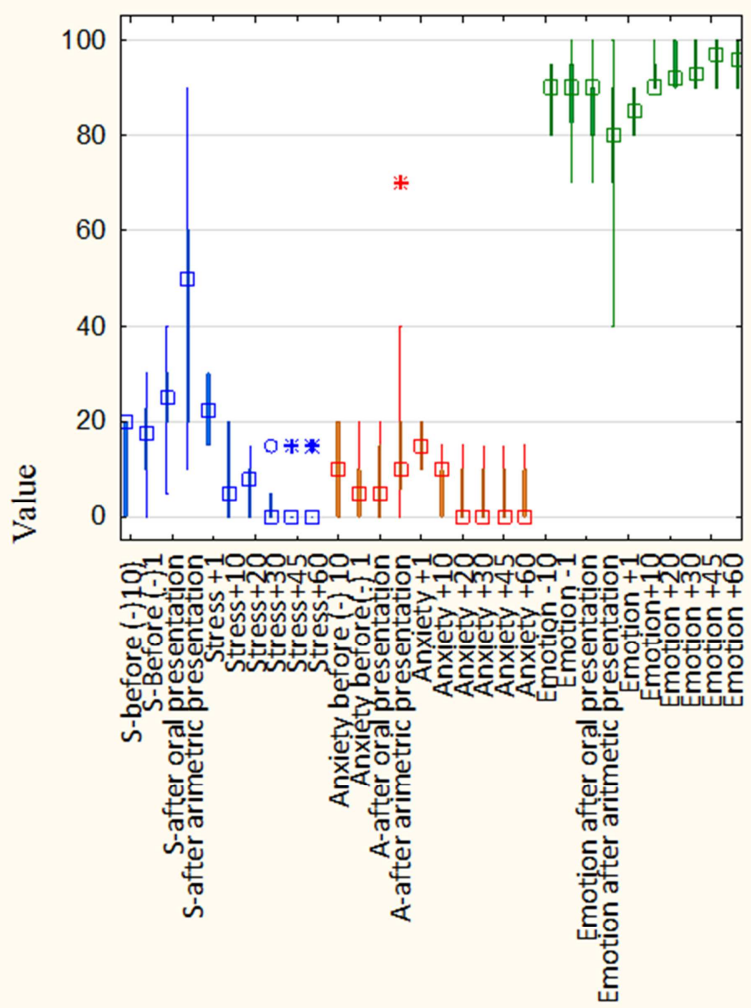

Timepoint
Type: Stress

a Type: Anxiety

Type: Emotion

- Outliers/Type: Stress

* Extremes/Type: Stress

* Extremes/Type: Anxiety

Figure 3. Perceived stress, anxiety and emotions before, during and after TSST participation. For perceived stress and anxiety, value " 0 " mean no, and "100" most intensive sense. For emotions, the scale is opposite," 0 " feel very bad. Values on the x-axis are given at each time point for cortisol samples before, during and up to 60 minutes after test. 


\section{Discussion}

\subsection{TSST}

Previous studies have shown that there is a difference between the cortisol response and the sympathetic adrenergic related response during and after the TSST [38, 39]. A study has shown that there is some adaptation to repeated TSSTs for the cortisol response but not for the sympathetic adrenergic response [39]. Therefore, we have only performed one test. Another fact is that the cortisol response, but not the sympathetic adrenergic response, depends on gender with a more increased response in men than women, menstrual cycle phase and ingestion of oral contraceptives with a more pronounced response in women during the luteal phase than during the follicular phase [38, 40]. This pronounced response in women is similar to the response in men [38]. Studies have found and reported gender differences in HPA axis response to stress and they found gender-specific associations between common glucocorticoid receptor gene variants and HPA- axis response to psychosocial stress [41, 42].

\subsection{Salivary Cortisol Response Versus TSST}

In the present study the first hypothesis was confirmed. The pattern of salivary cortisol for the whole group was consistent with previous results [31, 39] with an increase within 10-30 minutes after finishing the test. In the present study, three women with contraceptive implants completed the TSST. For the other three women without contraceptives we have no exact information about which point in time during the menstrual cycle the TSST was performed. We found no differences in response between these two groups of women. When comparing all these women's' responses with all men's' there was some difference with a more flattened cortisol response in women (not shown) in agreement with Kirschbaum et.al [38].

It is a small study but the results have a small distribution and obvious direction, so we believe that the results can be transferable and general. The most important finding for the TSST was a significant gender difference $(p=0.03)$ with higher salivary cortisol concentrations, but not for heart rate, about 10 minutes before the start (the anticipation phase) in women. The different cortisol responses in men and women during the TSST may reasonably be interpreted as follows: women going in to an unknown situation expect difficulties when performing in an unfamiliar setting. When they see that they can control the situation their perceived stress level drops. This interpretation is supported to some extent by Steptoe, \& Willemsen [43] who found that women experienced more stress than men if they had low job control. For men it seems to be the opposite: when going into an unknown situation they believe that they can perform but when they find out that they do not really do as well as they expected their perceived stress increase. Among men differences are seen at the individual level.

\subsection{The Heart Rate Response Versus TSST}

Normally, the heart rate increases during the test and decreases within ten minutes after the test to reach the basal original level within 25 minutes after the test [31, 38]. The heart rate increase during the test is constant and not significantly different after the interview compared to the arithmetic phase of the test [38]. This is in agreement with our results of a 15-to $20 \mathrm{bpm}$ increase during the test and a time interval to reach baseline level after the test of about 20 minutes without gender differences, Figure 1. In a previous study regarding physical effort it was observed that high physical effort led to increase in both heart rate and cortisol concentrations at group level [44]. The heart rate increase observed in the TSST could not be a consequence of physical effort because there was no one.

The second hypothesis that stress response was dependent on gender was confirmed but not for professional experience, education and age.

The third hypothesis, that there is a correlation between heart rate and salivary cortisol concentration, was not confirmed in the TSST. This is in agreement with a previous finding [39]. The hypothesis that heart rate might be able to replace cortisol as a method for measuring stress was not confirmed.

\subsection{Perceptions of Stress, Anxiety and Emotional Feelings During and After Test}

The perceived stress, anxiety and emotionally feelings changed fast after beginning of test and were recovered soon after end of test, similar to the heart rate response. These changes agree with previous results from Hellhammer \& Schubert [45].

\subsection{Theoretical Discussion}

This study rests primarily on the biomedical foundation because it is only biological markers that we have measured; we have measured biological response on stimuli (TSST). We have measured the biological response to stress stimuli via TSST as one way to investigate possible health problems.

We believe that our results show that it is difficult to lean on a single theory as an explanatory model when talking about health. We reckon that in order to get such a comprehensive answer, both at the individual and group level, a more interdisciplinary science is required, just like Eriksson's thoughts [46]. This is in line with Almeida Filho who is talking about a General Theory of Health [21, 47].

\subsection{Limitations}

\subsubsection{Strengths and Weaknesses}

The study has been well controlled. The study protocols for the TSST were followed completely and all participants completed the tests. All participants carried out the test in the same room under the same external physical conditions. Both 
salivary cortisol samples and heart rate measurements were complete, providing reliable results.

One weakness was that the group was small due to lack of financing laboratory costs. Another was that the number of women doing the test was too small for drawing firm conclusions based on gender differences.

A possible limitation may be the small number who was interested in participating and the selection was therefore too small. However, we consider this to be of less importance since physiological parameters were measured that participants could not influence.

\subsubsection{Validity, Reliability of the Instrument Used}

According to documentation from the manufacturer, the error for heart rate is $\pm 1 \%$ on the heart rate watches used which means that these values were highly reliable (personal contact). Salivary cortisol concentration is consistent and clearly linked to serum concentrations of cortisol, it is a safe and well-documented method to measure cortisol concentration and total intra- and inter-variability is about 10 percent $[48,49]$.

\subsection{Future Aspects}

In order to confirm the results that have emerged regarding the cortisol response in women at TSST a study is required with more participants and an equal proportion of both genders.

\section{Conclusions}

The TSST causes mental stress, and men and women exhibit significantly different patterns in terms of measured cortisol value when confronting unknown situations requiring certain standards of performance.

No correlation can be seen between heart rate and salivary cortisol concentration in these situations and they seem not to measure the same factors. Therefore, heart rate measurement cannot replace saliva cortisol in measurement of stress.

Perceived stress, anxiety, and emotional feelings are immediate and of short duration during the unfamiliar process.

In a future study it would be interesting to have a transdisciplinary approach. In connection with TSST, one could ask for the perceived stress either in a more detailed questionnaire or via an interview to capture various sides of the phenomenon.

\section{Generalizability of the results}

We have also performed a statistical analysis comparing the age, gender and educational profile for the personnel not participating in the study and there was no difference compared to the participants (table 1). Therefore, it seems that our results can be representative for a bigger group from the local and similar regions. Since we have not randomized the study group from all ambulance personnel in Sweden, who have very different working conditions, from country side to big cities we cannot conclude that all ambulance personnel react in this way. A bigger study population representative and randomized from all parts of Sweden must be performed to give such result.

\section{Ethics Approval and Consent to Participate}

Data collection followed the World Medical Association's principles in the Declaration of Helsinki [50]. Ethical permission for the study was obtained from the Regional Ethical Review Board in Gothenburg dated 25 June 2012 with Dno: 356-12. All participants were given both oral and written information. They gave their written consent to participate in the study and all were informed that they could end participation at any time without giving any reasons.

\section{Consent for Publication}

Not applicable.

\section{Availability of Data and Material}

The datasets used and/or analysed during the current study are available from the corresponding author on reasonable request.

\section{Competing Interests}

The authors declare that they have no competing interests.

\section{Funding}

The Research Fund at Skaraborg Hospital, Skövde, Sweden payed for analyses of salivary cortisol and gave time for collection and analysis of data for authors K. K and P. N. The views expressed in this article are those of the authors and not necessarily represent the views of the Fund.

\section{Authors' Contributions}

KK and PN; planning, implementation, statistics, preliminary script. AJ; planning, proof-reading. C-JT; planning, performing test, responsible for salivary cortisol analyses, statistics, figures, supervision of study.

\section{Acknowledgements}

We would like to thank biomedical scientist Margaret Bouveng-Käck for excellent processing of salivary cortisol samples. Thanks also to nurses Johan Ahlqvist, Carina Gustafsson and Brittmarie Kjellberg, and to psychologist Elisabeth Björklund and psychotherapist Birgitta Lannering for their participation as questioners during the TSST. We also thank biostatistician Salmir Nasic for help with statistical analysis. Finally, thanks to the participants. 


\section{Abbreviation}

$\mathrm{AUC}=$ area under the curve

$\mathrm{HRV}=$ heart rate variability

PHTLS $=$ prehospital trauma life support

PTSD $=$ post-traumatic stress disorder

TSST $=$ Trier Social Stress Test

\section{References}

[1] Socialstyrelsen, Socialstyrelsens föreskrifter (SOSFS 2009:10) om ambulanssjukvård 2009.

[2] Wiitavaara, B., et al., Striking a balance-health experiences of male ambulance personnel with musculoskeletal symptoms: A grounded theory. International journal of nursing studies, 2007. 44(5): p. 770-779.

[3] Nirel, N., et al., Stress, work overload, burnout, and satisfaction among paramedics in Israel. Prehospital and disaster medicine, 2008. 23(6): p. 537-546.

[4] Aasa, U., et al., Work - related psychosocial factors, worry about work conditions and health complaints among female and male ambulance personnel. Scandinavian journal of caring sciences, 2005. 19(3): p. 251-258.

[5] Jonsson, A. and K. Segesten, Daily stress and concept of self in Swedish ambulance personnel. Prehospital and disaster medicine, 2004. 19(3): p. 226-234.

[6] Sterud, T., Ø. Ekeberg, and E. Hem, Health status in the ambulance services: a systematic review. BMC Health Services Research, 2006. 6(1): p. 82.

[7] Maguire, B. J., et al., Occupational fatalities in emergency medical services: a hidden crisis. Annals of emergency medicine, 2002. 40(6): p. 625-632.

[8] Grønkjær, L. L., Nurses' experience of stress and burnout: a literature review. Klinisk Sygepleje, 2013. 27(01): p. 15-26.

[9] Yang, Y., et al., Salivary cortisol levels and work-related stress among emergency department nurses. Journal of Occupational and Environmental Medicine, 2001. 43(12): p. 1011-1018.

[10] Bounds, R., Factors affecting perceived stress in pre-hospital emergency medical services. Californian J Health Promot, 2006. 4(2): p. 113-131.

[11] Huang, Y., et al., Association between job strain and risk of incident stroke A meta-analysis. Neurology, 2015. 85(19): p. $1648-1654$

[12] Steptoe, A. and M. Kivimäki, Stress and cardiovascular disease: an update on current knowledge. Annual review of public health, 2013. 34: p. 337-354.

[13] Heikkilä, K., et al., Work stress and risk of cancer: metaanalysis of 5700 incident cancer events in 116000 European men and women. Bmj, 2013. 346: p. f165.

[14] Hegg-Deloye, S., et al., Current state of knowledge of posttraumatic stress, sleeping problems, obesity and cardiovascular disease in paramedics. Emerg Med J, 2014. 31(3): p. 242-247.

[15] Boudreaux, E., et al., Patient care and daily stress among emergency medical technicians. Prehospital and disaster medicine, 1996. 11(3): p. 188-193.

[16] Backé, E. M., et al., Assessment of salivary cortisol as stress marker in ambulance service personnel: comparison between shifts working on mobile intensive care unit and patient transport ambulance. International archives of occupational and environmental health, 2009. 82(9): p. 1057-1064.

[17] Millán, E. M., Occupational stress among health care professionals specialising in acute medicine. Emergencias, 2007. 19: p. 151-153.

[18] Berland, A., G. K. Natvig, and D. Gundersen, Patient safety and job-related stress: a focus group study. Intensive and critical care nursing, 2008. 24(2): p. 90-97.

[19] LeBlanc, V. R., et al., Paramedic performance in calculating drug dosages following stressful scenarios in a human patient simulator. Prehospital Emergency Care, 2005. 9(4): p. 439444.

[20] LeBlanc, V. R., et al., The impact of stress on paramedic performance during simulated critical events. Prehospital and disaster medicine, 2012. 27(4): p. 369-374.

[21] Almeida-Filho, N., Towards a Unified Theory of HealthDisease: I. Health as a complex model-object. Revista de Saúde Pública, 2013. 47: p. 433-450.

[22] Boorse, C., Health as a theoretical concept. Philosophy of science, 1977. 44(4): p. 542-573.

[23] Ereshefsky, M., Defining 'health'and 'disease'. Studies in History and Philosophy of Science Part C: Studies in History and Philosophy of Biological and Biomedical Sciences, 2009. 40(3): p. 221-227.

[24] Arman, M., et al., The Nordic tradition of caring science: the works of three theorists. Nursing science quarterly, 2015. 28(4): p. $288-296$.

[25] Eriksson, K., Hälsans idé.(2. uppl.) Stockholm: Liber. 1996, Almqvist \& Wiksell medicin.

[26] Eriksson, K., Den lidande människan. 1994: Liber utbildning.

[27] Segesten, K. and K. Dahlberg, Hälsa och vårdande: I teori och praxis. Stockholm: Natur \& kultur, 2010.

[28] Hellhammer, D. H., S. Wüst, and B. M. Kudielka, Salivary cortisol as a biomarker in stress research. Psychoneuroendocrinology, 2009. 34(2): p. 163-171.

[29] Törnhage, C.-J., Salivary cortisol for assessment of hypothalamic-pituitary-adrenal axis function. Neuroimmunomodulation, 2009. 16(5): p. 284-289.

[30] Karlsson, K., P. Niemelä, and A. Jonsson, Heart rate as a marker of stress in ambulance personnel: a pilot study of the body's response to the ambulance alarm. Prehospital and disaster medicine, 2011. 26(1): p. 21-26.

[31] Kirschbaum, C., K.-M. Pirke, and D. H. Hellhammer, The 'Trier Social Stress Test'-a tool for investigating psychobiological stress responses in a laboratory setting. Neuropsychobiology, 1993. 28(1-2): p. 76-81.

[32] Het, S., et al., Neuroendocrine and psychometric evaluation of a placebo version of the 'Trier Social Stress Test'. Psychoneuroendocrinology, 2009. 34(7): p. 1075-1086. 
[33] Von Dawans, B., C. Kirschbaum, and M. Heinrichs, The Trier Social Stress Test for Groups (TSST-G): A new research tool for controlled simultaneous social stress exposure in a group format. Psychoneuroendocrinology, 2011. 36(4): p. 514-522.

[34] Jönsson, P., et al., Cardiovascular and cortisol reactivity and habituation to a virtual reality version of the Trier Social Stress Test: a pilot study. Psychoneuroendocrinology, 2010. 35(9): p. 1397-1403.

[35] Törnhage, C.-J., Reference values for morning salivary cortisol concentrations in healthy school-aged children. Journal of Pediatric Endocrinology and Metabolism, 2002. 15(2): p. 197-204.

[36] Pruessner, J. C., et al., Two formulas for computation of the area under the curve represent measures of total hormone concentration versus time-dependent change. Psychoneuroendocrinology, 2003. 28(7): p. 916-931.

[37] Fekedulegn, D. B., et al., Area under the curve and other summary indicators of repeated waking cortisol measurements. Psychosomatic medicine, 2007. 69(7): p. 651-659.

[38] Kirschbaum, C., et al., Impact of gender, menstrual cycle phase, and oral contraceptives on the activity of the hypothalamus-pituitary-adrenal axis. Psychosomatic medicine, 1999. 61(2): p. 154-162.

[39] Schommer, N. C., D. H. Hellhammer, and C. Kirschbaum, Dissociation between reactivity of the hypothalamus-pituitaryadrenal axis and the sympathetic-adrenal-medullary system to repeated psychosocial stress. Psychosomatic medicine, 2003. 65(3): p. 450-460.

[40] Reschke - Hernández, A. E., et al., Sex and stress: Men and women show different cortisol responses to psychological stress induced by the Trier social stress test and the Iowa singing social stress test. Journal of neuroscience research, 2017. 95(1-2): p. 106-114.

[41] Kudielka, B. M. and C. Kirschbaum, Sex differences in HPA axis responses to stress: a review. Biological psychology, 2005. 69(1): p. 113-132.

[42] Kumsta, R., et al., Sex specific associations between common glucocorticoid receptor gene variants and hypothalamuspituitary-adrenal axis responses to psychosocial stress. Biological psychiatry, 2007. 62(8): p. 863-869.

[43] Steptoe, A. and G. Willemsen, The influence of low job control on ambulatory blood pressure and perceived stress over the working day in men and women from the Whitehall II cohort. Journal of hypertension, 2004. 22(5): p. 915-920.

[44] Karlsson, K. J., et al., Using shoulder straps decreases heart rate variability and salivary cortisol concentration in Swedish ambulance personnel. Safety and health at work, 2016. 7(1): p. 32-37.

[45] Hellhammer, J. and M. Schubert, The physiological response to Trier Social Stress Test relates to subjective measures of stress during but not before or after the test. Psychoneuroendocrinology, 2012. 37(1): p. 119-124.

[46] Eriksson, K., Caring science in a new key. Nursing science quarterly, 2002. 15(1): p. 61-65.

[47] Almeida Filho, N. d., For a general theory of health: preliminary epistemological and anthropological notes. Cadernos de Saúde Pública, 2001. 17: p. 753-770.

[48] Törnhage, C. J. and G. Alfvén, Children with recurrent psychosomatic abdominal pain display increased morning salivary cortisol and high serum cortisol concentrations. Acta Paediatrica, 2015. 104(12).

[49] Dorn, L. D., et al., Salivary cortisol reflects serum cortisol: analysis of circadian profiles. Annals of clinical biochemistry, 2007. 44(3): p. 281-284.

[50] Puri, K., et al., Declaration of Helsinki, 2008: implications for stakeholders in research. Journal of postgraduate medicine, 2009. 55(2): p. 131. 